\section{WHAT WERE THE REVIEWERS}

THINKING?

To the editor: It seemed a curious choice to publish the peer-reviewed article "Feasibility and Speed of Insertion of Seven Supraglottic Airway Devices under Simulated Airway Conditions" by Robak and colleagues $^{1}$ in the November 2012 issue of the CFEM. The article purports to compare the speed of insertion of seven different supraglottic airway devices by inexperienced medical students using one type of airway manikin. The authors simulated one "physiologic" and five "pathologic" conditions. They trained 45 "inexperienced" medical students for only 20 seconds per device. This was justified because they "considered the use of the airway devices self-explanatory." They chose average speed of insertion as the variable to report rather than the rate of success in placement of the device within a clinically relevant time interval. The average speed of placement of the devices in the simulations was reported in hundreds of a second, similar to a downhill ski race. The average speed of insertion on the first attempt under physiologic conditions varied among devices from 4.64 to 7.49 seconds. It varied among devices in the first attempt during simulated tongue edema from 4.82 to 15.31 seconds. The authors reported that students were unable to insert five of the seven airway devices in other simulated "pathologic" conditions, such as trismus or limited mobility of the cervical spine. It is hard to believe that all 45 students failed to place any of five devices in other simulated pathologic conditions but were able to place the remaining two devices under these conditions. The two devices that could be placed, the Easy Tube and the Combitube, were able to be inserted on average in just 4.82 and 6.24 seconds, respectively, during the first attempt. Interestingly, the Combitube was the only device inserted on average faster under "pathologic" conditions than under "physiologic" conditions during the first attempt.

The authors "observed a significant difference between the various supraglottic airway devices with regard to speed of insertion." However, statistical significance does not always equate to clinical significance. The authors did not appear to appreciate this. They also noted that "the manikin's anatomy may favour a specific model of airway device." ${ }^{\prime}$ Despite this, and presumably based on their results, they go on to conclude that "the Combitube and Easy Tube may offer advantages in difficult airway situations." "may." However, before replacing your supraglottic airways, note that one of the authors was reported to "have received royalties from Covidien in the past." ${ }^{1}$ Guess what device Covidien manufactures and distributes? You guessed it: Combitubes!

Don Eby, MD, MSc, CCFP(EM) Owen Sound, ON

\section{Reference}

1. Robak O, Leonardelli M, ZedwitzLiebenstein K, et al. Feasibility and speed of insertion of seven supraglottic airway devices under simulated airway conditions. CFEM 2012;14:330-4. 